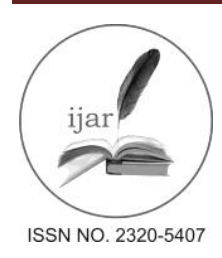

Journal homepage: http://www.journalijar.com

Journal DOI: 10.21474/IJAR01

RESEARCH ARTICLE

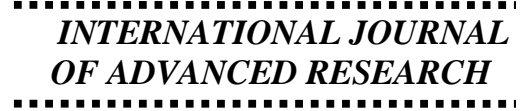

"

\title{
A CASE REPORT : HEPATIC HYDATID CYST WITH RENAL INVOLVEMENT.
}

V.S. Rathod ${ }^{1}$, Kasturi ${ }^{2}$, ${ }^{*}$ Shuvankar Mukhopadhyay ${ }^{3}$, RohitSinha ${ }^{4}$, Tamboli S $^{5}$, V Shegokar ${ }^{6}$.

1. Associate Professor, Microbiology Department, Dr SC ChavanGov Medical College, Nanded,Maharashtra.

2. Resident,Microbiology Department, Dr SC ChavanGov Medical College, Nanded,Maharashtra.

3. Assistant Professor. Microbiology Department, Dr SC ChavanGov Medical College, Nanded,Maharashtra.

4. Resident,Microbiology Department, Dr SC ChavanGov Medical College, Nanded,Maharashtra.

5. Associate Professor, Microbiology Department, Dr SC ChavanGov Medical College, Nanded,Maharashtra.

6. Professor\& HOD, Microbiology Department, Dr SC ChavanGov Medical College, Nanded,Maharashtra.

\section{Manuscript Info}

Manuscript History:

Received: 15 April 2016

Final Accepted: 29 May 2016

Published Online: June 2016

Key words:

Hydatid cyst, liver, kidney.

*Corresponding Author

Shuvankar

Mukhopadhyay.

\begin{abstract}
Hydatid disease is a parasitic infection by a tape worm of the Genus Echinococcus. It effects human as well as other mammals like; sheep, dogs, rodents and horses. Hydatid cyst is one of the known cause of Liver mass. Investigations and appropriate management provides improved quality of life and substantially decrease mortality ratio. We are presenting here a case of Hydatid cyst in right lobe of Liver with retroperitoneal extension into right kidney So right lobectomy with enbloc hydatid cyst resection was performed at tertiary care hospital in peripheral Maharashtra A 40 years old female residing in District Nanded, presented with intermittent abdominal pain for 2 years. Abdominal sonography showed a multiloculated cystic mass in the right lobe of liver with some punctuate calcifications at the peripheral wall and thickened septa.Cyst was also found in kidney Under the impression of hepatic hydatid cyst, the patient received right lobectomy with en block cyst removal from liver and right kidney Histological study of the lesion showed a cyst with many daughter cysts lying free in the cyst fluid. Hydatid cyst may be asymptomatic or may lead to lethal complications. Surgery is considered the optimal treatment that has the potential to remove the cyst and leads to complete cure. It is important to make a preoperative diagnosis based on the typical image findings, so that surgeons may take particular precaution not to rupture the lesion as peritoneal spillage may lead to disseminated implantation.and anaphylaxis.
\end{abstract}

Copy Right, IJAR, 2016,. All rights reserved.

\section{Introduction:-}

Hydatid disease (HD) is a helminthic anthropozoonosis with worldwide distribution, caused by the larval stage of the Echinococcus tapeworm. It can occur almost anywhere in the body with a variety of imaging features, which may change according to the growth stage, associated complications and affected tissues. ${ }^{1}$

A definitive diagnosis requires a combination of imaging,microscopic and serologic studies. Hepatic hydatid cysts can be treated by surgery, chemotherapy and/or percutaneous aspiration. Although a number of scolecidal agents have been developed against HD, surgical resection is the only curative treatment. HD primarily affects the liver and demonstrates characteristic imaging findings on ultrasonography (US); computed tomography (CT) and magnetic resonance imaging (MRI) are highly accurate in detecting hepatic HD.

However, hepatic hydatid cysts in an unusual location or of an unusual dimension, with atypical imaging findings, may complicate the differential diagnosis. Large cysts, today fairly rare even in endemic areas, are called giant 
hydatid cysts (GHCs) . GHCs have exophytic growth through the natural routes provided by the liver capsule, ligaments and peritoneum. In these cases, MRI is the most useful tool among the imaging techniques to demonstrate cyst migration.

We describe an unusual case of a GHC originating from the right lobe of the liver with exophytic growth into the right kidney. This atypical growth raised doubts about the most appropriate surgical approach. MRI easily clarified the origin of the cyst as the liver, allowing accurate surgical planning. ${ }^{1}$

\section{Case report:-}

A forty year old female presented with intermitted upper abdominal pain for two years. She is a Villager from nanded district and there was history of weight loss and off/on history of fever. There was no previous hospitalization and surgical interventions. No significant family history could be found. There was history of keeping dog at home. On clinical examination patient was a fibrile vital were stable, systematic review was normal.

Abdominal Examination: 5.1X5.2 cm mass firm in consistency in Epigastric area moves with respiration slight tenderness in epigastric area. There were no visible pulsation or penstaltic movements on it. USG abdomen CT. Abdomen were both in favour of Hydatid cyst involving right lobe of Liver According to clinical history and image finding Hydatid cyst was suspected. The patient was admitted in surgery department for further treatment.Physical examination was generally normal. The data of CBC and serum biochemistry was unremarkable.except mild eosinophilia. The serological test including the hydatidimmunoelectrophoresis, enzyme-linkedimmunosorbent assay (ELISA), latex agglutination and indirect haemagglutination (IHA) test were not performed during admission. Her stool was soft and brown and no parasites were found. Abdominal sonography showed a complex cystic lision with solid component in right lobe of liver and in right kidney. Patient prepared forExploratory Laparotomy, fitness for General Anesthesia confirmed. Upper mid line with extension into right hypochondrium Incision made.enbloc resection of cyst mass was performed.Hydatid sand was send to Microbiology Department and was examined by wet mount preparation and also LPCB mount. Both mounts revealed scolex and hooklet form and also brood capsule with Daughter cyst of Echinococcus species. (Fig 1,2,3)

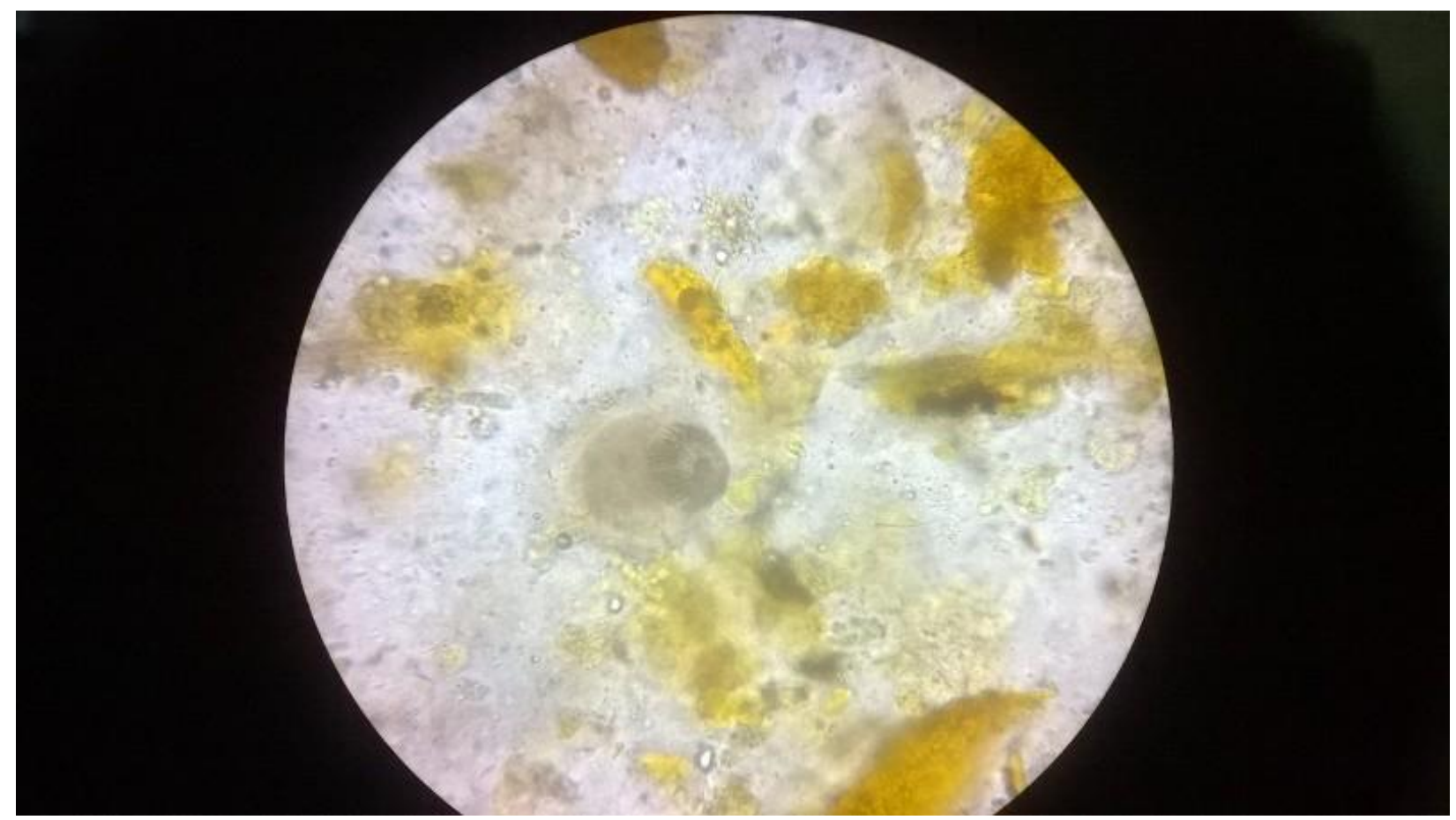

Figure 1:- Scolex and Hooklet in wet mount of Hydatid sand (100× Magnification) 


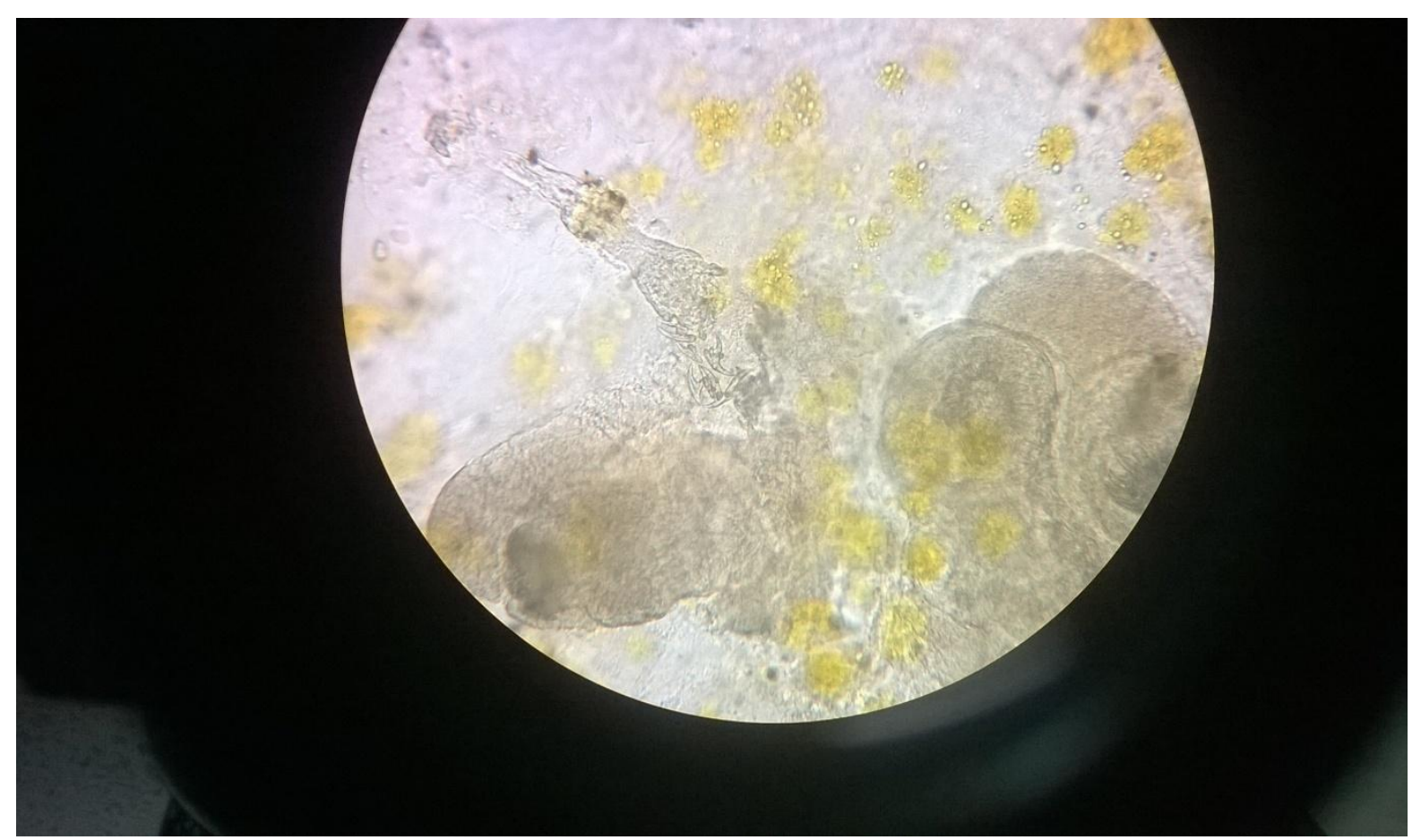

Figure 2:- hooklet in wet mount(100× Magnification)

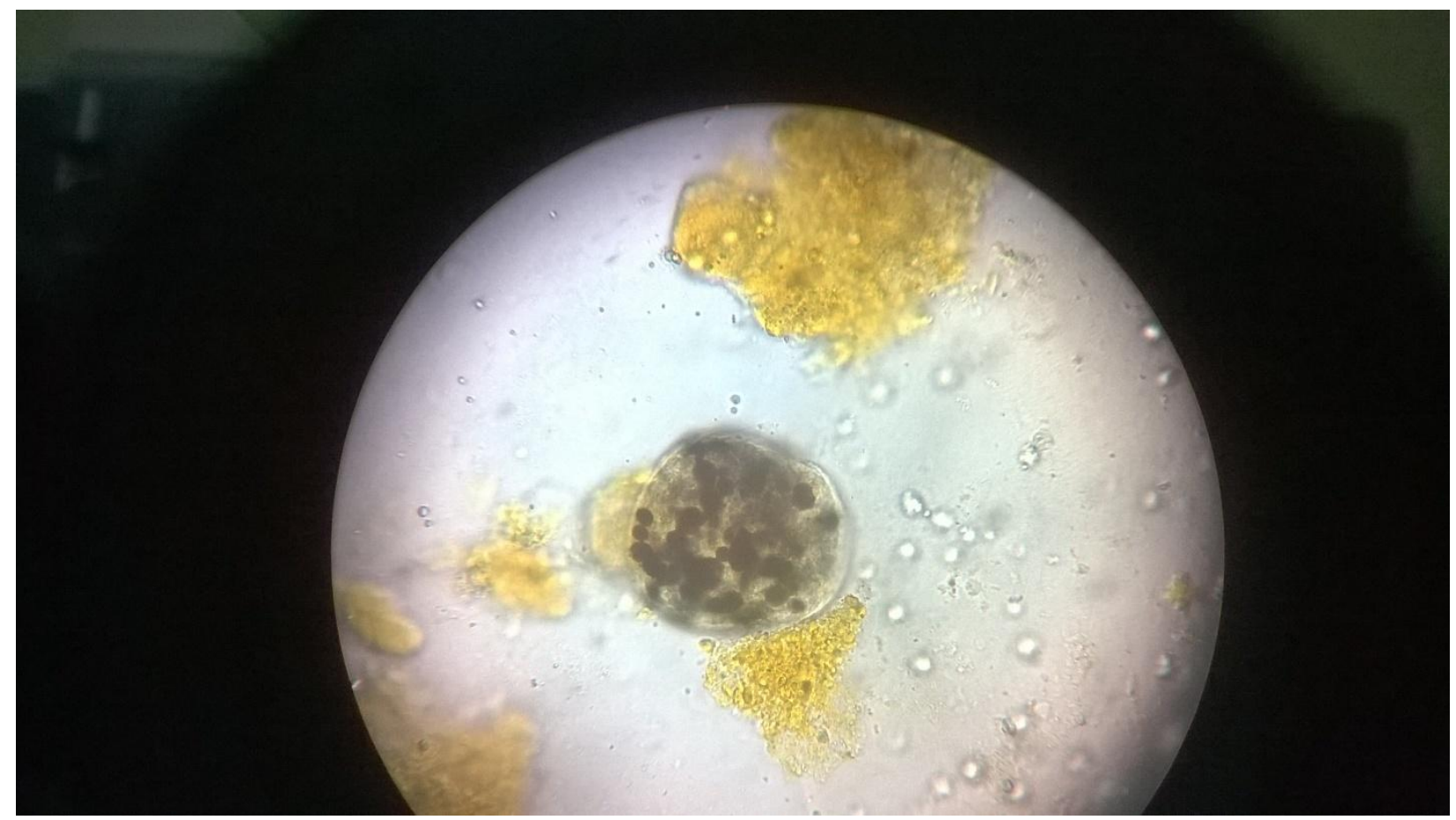

Figure 3:- Brood capsule and daughter cyst in wet mount of Hydatid sand(100× Magnification)

\section{Discussion:-}

Hydatid cyst is a zoonotic disease that occurs throughout the world particularly in those areas where people are involved in cattle rearing profession ${ }^{2}$. Usually two types of Echinococcus infections are Echinococcus granulosus and Echinococcus multicularis. In human this cyst is caused by the larva of a tapewormEchinococcus granulosus ${ }^{3}$, whereas Echinococcus multicularisis not common but more invasive by mimicking a malignancy ${ }^{4}$. 
The life cycle of E. granulosus alternates between carnivores and herbivores for instance dogs and sheep, whereas man is an accidental intermediate host with ending point in parasite's life cycle. The liberated ova burrow through intestinal mucosa and are carried by the portal vein to the liver, where they develop into adult cysts. Liver is most common site of Hydatid disease and most cysts are located in the right lobe ${ }^{4}$. Some ova pass through capillary sieve and become lodged in any part of the body. The bloodstream reaches, including the lung, peritoneum, kidney, brain, mediastinum, heart, bone, soft tissues and most of the other body parts ${ }^{5,6}$.

Some times Hydatid cyst may be remained asymptomatic for years long. Its presence may become evident when the hepatomagely is found or a cystic lesion is noted when the liver is imaged for other reasons. It may be painful or lead to complications such as rupture into biliary tract or peritoneal cavity which may cause cholangitis or anaphylactic shock ${ }^{7}$.

Hydatid cyst can be solitary or multiple in nature. Imaging findings depends upon the stage of cyst growth. Laboratory screening examination in most cases showed normal results. The diagnosis can be confirmed when imaging is combined with serological tests. Here in mentioned case serological tests were not available. Calcification is usually curlivilinear or ring like and involves the pericyst. It is seen on radiography that, there are 20-30 \% cases are of hepatic hydatid cyst. The presence of multiple echogenic foci that fall into the dependent portion of the cyst during posture change of the patient is a characteristic ultrasound finding such as snowstorm sign. On CT scan and MRI the septa and cyst wall frequently enhance after injection of contrast material frequently. A low signal intensity rim that is more evident on $\mathrm{T} 2$ weighted images has been described as characteristic of hydatid cyst as opposed to nonparasitic cyst in Liver \& Lungs. In a few equivocal cases with -ve serological tests an image guided aspiration of the cyst contents for microscopic analysis can help to establish a definitive diagnosis prior to therapy ${ }^{8}$. However spillage of contents should avoided during the procedure.

Here in our case abdominal sonography showed daughter cysts separated by solid components which should be hydatid matrix containing broken cysts, scolices and hydatid sand. The appearance of hydatid matrix in CT be variable depending upon the content that fills the cystic mass and the small cyst here within. There may be so-called 'cyst in cyst' appearance. The deferential diagnosis can be made when there is history of living in endemic regions along with these imaging findings.

The patient with hydatid cyst frequently present a therapeutic challenges. Medical treatment of hydatid cyst with drugs like mebendazole/ albendazole has been reported but medical therapy alone has controversial results 9 . It has been used in the prevention of post-operative local recurrence and sterilization before surgery ${ }^{10}$. Percutaneous drainage had been proposed as an alternative to surgery especially for the patients who don't want to undergo surgical procedure ${ }^{11}$. Surgery is considered as best possible treatment having potential to remove the cyst and complete cure ${ }^{12}$. The main principle of surgical treatment is to eradicate the parasite, prevent intra-operative spillage of contents and obliterate the residual cavity. Recurrence of hydatid cyst may occur either from spillage of hydatid fluid during the surgical procedure or from any re-infestation of the patient ${ }^{13}$ our case mentioned above was rare in this sense cyst were found not only in liver but also in kidney although no renal manifestation was evident in patient.

Hydatid cyst of liver due to E. granulosus infection remained challenging clinical problem throughout the world. However it is matter of very importance to kept in mind that; when a cyst lesion is encountered anywhere in the body, diagnosis can be improved by making familiarity with imaging features, especially for the patients living in countries where this disease is endemic. 


\section{References:-}

1. Text book of Parasitology by S. C, Parija $2^{\text {nd }}$ edition page 134-135 Elesvier

2. Agaoglu N, Turkyilmaz S, Arslan MK. Surgical treatment of hydatid cysts of the liver. Br J Surg 2003; 90:1536-1541

3. Kumar MJ, Toe K, BanerjeeRD.Hydatid cyst of liver. Postgrad Med J 2003; 79: 113-114

4. Polat P, Kantarci M, Aiper F, Suma S, Koruyucu MB, Okur A. Hydatid disease from head to toe. Radiographics

5. 2003; 23: 475-494

6. Lewall DB, McCorkell SJ. Hepatic echinococcal cysts: sonographic appearance and classification. Radiology 1985;155: 773-775

7. Von Sinner W, Te Strake L, Clark D, Sharif H. MR imaging in hydatid disease. Am J Roentgenol 1991; 157:741-745

8. Lygidakis NJ. Diagnosis and treatment of intrabiliary rupture of hydatid cyst of the liver.Arch Surg 1983; 118: 1186- 1189

9. Haddad MC, Al-Awar G, Huwaijah SH, Al-Kutoubi AO. Echinococcal cysts of the liver: a retrospective analysis of clinic-radiological findings and different therapeutic modalities. Clin Imaging 2001; 25: 403-408

10. Todorov T, Vutova K, Mechkov G, Petkov D, Nedelkov G, Tonchev Z. Evaluation of response to chemotherapy of human cystic echinococcus. Br J Radiol. 1990; 63: 523-531

11. Topcu S, Kurul IC, Tastepe I, Bozkurt D, Gulhan E, Cetin G. Surgical treatment of pulmonary hydatid cysts in children. J Thoraccardiovasc Surg. 2000; 120: 1097-1101

12. Akhan O, Ozmen MN. Percutaneous treatment of liver hydatid cysts.Eur J Radiol. 1999; 32: 76-85

13. WHO Guidelines for treatment of cystic and alveolar echinococcosis in humans. Bull World Health Organ. 1996; 74: 231-242

14. Ustunsoz B, Akhan O, Kamilogu MA, Somunca I, Ugurel MS, Cetiner S. Percutaneous treatment of hydatid cysts of the liver: long-term results. Am J Roentgenol 1999; 172: 91-96 